\title{
PRIORITY ACCESS SERVICE IN CELLULAR AND PCS NETWORKS
}

\author{
Christopher Redding \\ Institute for Telecommunication Sciences \\ Boulder, $\mathrm{CO}$ \\ Carol-Lyn Taylor \\ National Communications System \\ Arlington, VA
}

\begin{abstract}
Wireless telephony can greatly assist National Security and Emergency Preparedness (NS/EP) personnel in providing emergency communications. With the widespread availability of wireless telephony, a cost effective means of communication is readily available to NS/EP users. However, due to the heavy traffic demand placed upon existing or surviving systems in the aftermath of a disaster, severe network congestion will result in high call blocking to NS/EP users when their services are needed the most. This need has resulted in the wireless intelligent network (WIN) implementation of a priority access service (PAS) development effort. The WIN implementation of PAS provides a means for NS/EP users to obtain priority access on the next available radio channel when network congestion is encountered.
\end{abstract}

\section{INTRODUCTION}

Wireless telephony has grown dramatically since its inception in the early 1980s. Wireless subscribers now enjoy the nearly ubiquitous availability of seamless, economical wireless access throughout the country. As a result, wireless subscribers have become more dependent on wireless communications, and more insistent on features that provide value-added functionality to their basic service. Likewise, subscribers in the Government have also become dependent on wireless communications in the performance of their mission, in particular for national security and emergency preparedness (NS/EP) communications. NS/EP responders frequently provide communications in a mobile environment and require wireless connectivity when responding to emergencies such as earthquakes, hurricanes, floods, etc. Commercial wireless service provides a cost-effective capability for NS/EP communications that leverages the use of the public switched telephone network (PSTN) versus the costly development of a private network. This minimizes the development costs and deployment time of such a capability, as well as providing the broadest accessibility and furnishing NS/EP responders with crucial PSTN access. However, due to heavy traffic demand placed upon surviving systems in the aftermath of a disaster, severe network congestion will result in high call blocking in wireless and wireline communications to the critical disaster relief officials when their services are needed the most. Consequently, a priority access mechanism that will provide NS/EP responders with the next available channel is needed. A wireline priority access capability currently exists in the Government Emergency Telecommunications Service (GETS) program, but a wireless capability is needed to extend that capability into the wireless domain.

\section{BACKGROUND}

The priority access service (PAS) program was developed by the Federal Communications Commission (FCC) and is managed by the Office of the Manager, National Communications System (OMNCS). OMNCS is a Federal Government organization established to perform NS/EP telecommunications functions among its 23 member organizations. A primary mission of OMNCS is to develop a responsive, survivable, and enduring national telecommunications infrastructure to support NS/EP telecommunications needs of the Federal Government. As a first step in satisfying this mission requirement, OMNCS developed and deployed GETS. GETS is designed and maintained in a state of readiness that makes use of available PSTN resources, should outages occur during an emergency or crisis. GETS is accessed through a universal access number and a PIN using standard telephone equipment. Priority treatment of calls is provided by the high probability of completion (HPC) Call Indicator codepoint in the Signaling System Number 7 (SS7) Initial Address Message (IAM) Calling Party Category (CPC) field. HPC is standardized in American National Standard ANSI T1.6311993 [1]. HPC IAMs are set to a congestion priority of 1 , while normal traffic has a value of 0 . When the HPC codepoint is given a priority of 1 , call blocking is significantly decreased when SS7 network congestion is encountered [2]. 


\section{POLICY}

The FCC issued a Second Report and Order [3] on July 3, 2000 that establishes the regulatory, administrative, and operational framework that enables commercial mobile radio service (CMRS) providers to offer PAS to NS/EP personnel. CMRS providers include cellular licensees, personal communications service (PCS) licensees, specialized mobile radio (SMR) licensees and others (satellite, air-to-ground). The FCC rules do not require CMRS providers to implement PAS, they merely permit service providers to offer PAS to public safety personnel at the Federal, State and local levels to help meet the NS/EP needs of the Nation.

The FCC requires service providers to adhere to a set of uniform operating protocols concerning the number of priority levels and their associated NS/EP users. Accordingly, PAS will provide 5 levels of priority as shown in Table 1, with each level having a distinct class of user. Commercial applications are also being explored, although they will not fall into any of the NS/EP levels in Table 1.

Table 1. Priority Level and Associated NS/EP Users

\begin{tabular}{|c|l|}
\hline Priority Level & \multicolumn{1}{|c|}{ NS/EP User } \\
\hline 1 & Executive Leadership and Policy Makers \\
\hline 2 & $\begin{array}{l}\text { Disaster Response, Military Command } \\
\text { and Control }\end{array}$ \\
\hline 3 & $\begin{array}{l}\text { Public Health, Safety, and Law } \\
\text { Enforcement Command }\end{array}$ \\
\hline 4 & $\begin{array}{l}\text { Public Services, Utilities and Public } \\
\text { Welfare }\end{array}$ \\
\hline 5 & Disaster Recovery \\
\hline
\end{tabular}

\section{PROGRAM GOALS}

The overall goals of PAS are that the service should 1) provide access using commercially available mobile sets in areas where PAS is offered, 2) transparently operate in the system, 3) be interoperable among wireless infrastructures and available uniformly across the nation, 4) perform effectively under conditions of up to eight times call overload and provide a PAS user a high probability of radio channel access at the origination or termination cell site of $90 \%$ or greater, 5) perform effectively regardless of origination or termination location, and 6) should ensure service continuity during handoff and roaming conditions to the extent technically and economically feasible.

\section{PRIORITY ACCESS SERVICE}

The PAS capability leverages the use of existing commercial wireless networks to provide NS/EP users priority treatment of calls in the event that network congestion is blocking call attempts. PAS provides the means for qualified and authorized NS/EP users to obtain priority access to the next available radio channel in a wireless call path when emergency calls are placed. PAS is activated on a per call basis and is capable of mobile-to-wireline, wireline-tomobile and mobile-to-mobile links.

When an NS/EP user places a PAS call and the service is activated, the mobile set requests a radio channel via the control channel messaging. In non-congested environments a radio channel is allocated to the mobile set and the call is connected. In congested environments a radio channel may not be available, so the network will not be able to grant one. In this situation PAS is invoked. Conversely, when network congestion is not present, PAS is not activated. When congestion is encountered at call origination, the NS/EP caller is placed in a queue and will be given the next available radio channel. PAS does not preempt calls in progress and is to be used in emergency situations where network congestion is blocking call attempts.

PAS can be implemented over CMRS networks to include cellular, PCS, SMR and other commercial wireless technologies as ascertained by the FCC. Per the FCC Second Report and Order the OMNCS is tasked with managing the PAS program. As PAS evolves there may be several implementations that are proliferated. One particular solution that is currently being pursued employs the wireless intelligent network (WIN) for operation in cellular and PCS networks, which is the focus of this paper. This approach involves a standardized WIN application, which will provide interoperability among different service provider implementations.

\section{WIN IMPLEMENTATION OF PAS}

One of several potential PAS implementations, the WIN implementation employs a standards-based WIN architecture. WIN [4] is the primary result of the telephone industry's desire to offer improved services to their wireless subscribers. WIN builds on existing wireless architectures, which enables a graceful evolution to an intelligent network without making current wireless infrastructures obsolete. WIN describes a network architecture that provides flexible service creation, service management/control for wireless 
network operators, and rapid deployment of enhanced subscriber services. Examples of WIN services include radio controlled services, incoming call screening, prepaid charging, and global roaming. OMNCS is pursuing a standardized WIN implementation of PAS via the Telecommunications Industry Association (TIA) and the $3^{\text {rd }}$ Generation Partnership Project 2 (3GPP2) standards bodies.

\section{PAS OPERATION}

This section presents the current concept of a PAS implementation. Note that it is currently in the development stage; hence, it is subject to change. A PAS call is placed like a normal wireless call, using a mobile set with connectivity to the PSTN made via a base station controller (BSC) and mobile switching center (MSC). PAS calls encountering a "No Radio Available" condition in a cell are queued and processed for the next available channel in the cell. This process is managed via a WIN SCP (see Figure 1), through a signal transfer point (STP) in the network. A queued call is processed by the originating MSC based on instructions from the WIN SCP. It is envisioned that the PAS calling party authorization and priority level determination at call origination are made by a check in the PAS field in a database maintained in the WIN SCP, or as an alternative via a Home Location Register (HLR) or other appropriate platform.

When an authorized user invokes PAS at call origination and a radio channel is not available, the call is queued for the next available radio channel in the cell in accordance with the user's priority level and call initiation time. When a radio channel becomes available, it is assigned to the call, the user is alerted, and the call is connected. In a non-congested environment PAS is not invoked. In the event when PAS is invoked and 1) a radio channel is not available, 2) the queue for the cell is full, and 3) the user's PAS priority is higher than one or more PAS calls already in the queue; then the lowest and most recent call in the queue is dropped from the queue. The user's call is placed in the queue in accordance with the user's priority level and call initiation time. The user should be given an indication that the request has been queued. When a radio channel becomes available and is assigned to the call, the user is alerted and the call proceeds as normal. If a PAS caller moves to a new cell while still in queue for a radio channel in the original cell, two scenarios are possible depending on the availability of a radio channel in the new cell: a) if a radio channel is available in the new cell, the user is alerted and the call proceeds as normal in the new cell and is dropped from the queue for the original cell;

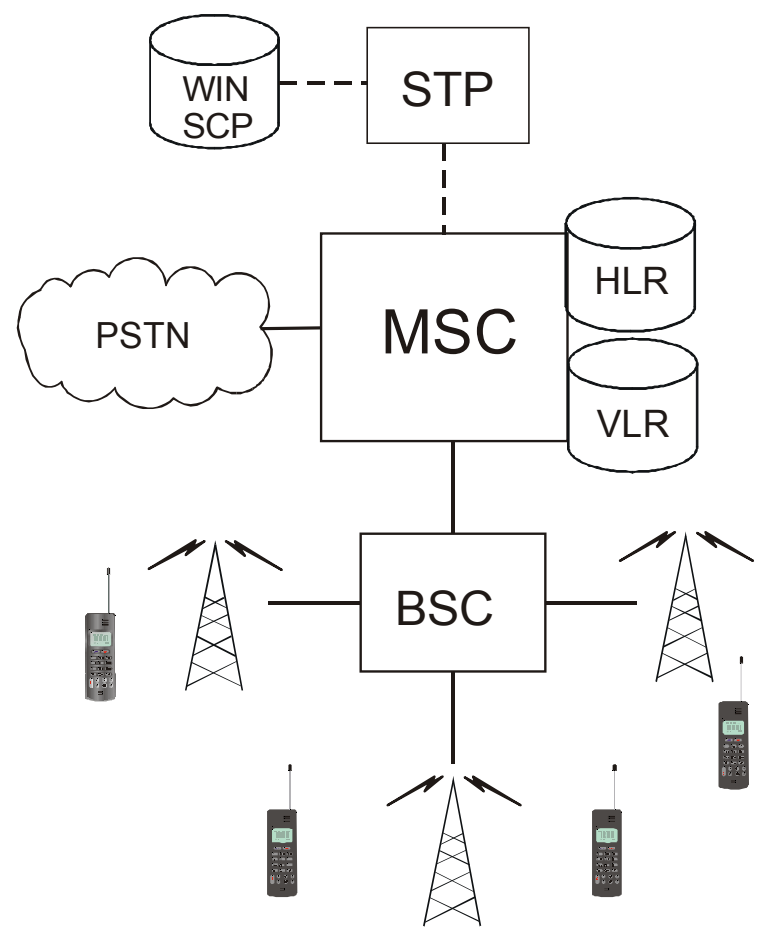

Figure 1. WIN architecture.

or b) if a radio channel is not available in the new cell, the call is queued for the next available radio in the new cell in accordance with the user's priority level and call initiation time, and the call is dropped from the queue in the original cell. When a radio channel becomes available in the new cell and is assigned to the call, the user is alerted and the call proceeds as normal in the new cell.

For the situation of call termination to a mobile user, the call proceeds as normal when a PAS call has been setup to the termination MSC and an egress radio channel is available. If an egress radio channel is not available, the call is queued for the next available radio channel in the cell serving the called party in accordance with the call's priority level. The calling party should be given an indication that the request has been queued. When an egress radio channel becomes available and is assigned to the call, the call proceeds as normal. In the event when PAS is invoked and 1) an egress radio channel is not available, 2) the queue for the cell serving the called party is full, and 3) the call's priority level is higher than one or more PAS calls in the queue; then the lowest, most recent call in the queue is dropped from the queue. The user's call is entered in the queue in accordance with the call's priority. When an egress radio channel becomes available and is assigned to the call, the call proceeds as normal. If the called party moves to a new terminating cell while the user's (calling party's) call is still in queue at the termination MSC for the called party in the 
original terminating cell, two scenarios can occur: a) if an egress radio channel is available in the new terminating cell serving the called party, the call proceeds as normal in the new terminating cell and the call request is dropped from the queue for the original terminating cell; or b) if an egress radio channel is not available in the new terminating cell for the called party, the call is queued for the next available radio in the new terminating cell in accordance with the call's priority level, and the call request is dropped from the queue in the original terminating cell. When an egress radio channel becomes available in the new terminating cell and is assigned to the call, the call proceeds as normal in the new terminating cell.

\section{ENHANCEMENTS TO WIN}

The WIN implementation of PAS is in the development stage; hence the WIN enhancements described in this section are subject to change. This section presents the current enhancements that will be needed to provide PAS functionality. The WIN Basic Call State Model (BCSM) provides a high level vendor and implementation independent view of the call and connection processing in the MSC. The BCSM has two primary components, the Points in Call (PIC) and Detection Point (DP) for Origination and Terminating halves of the call. A PIC is required to meet the basic call and connection states for the WIN service logic. A DP is a point at which transfer to WIN service logic can occur.

On the Origination half of the BCSM, the addition of a new Origination_Denied DP and a new No_Radio_Available trigger in the DP enables a WIN SCP to exercise the new Request Radio Channel capability.

When no radio channels are available for a call termination, the termination is denied and basic call and connection processing causes a transition to the T-Busy DP. On the Terminating half of the BCSM, the addition of a new No_Radio_Available trigger to a modified T-Busy DP to process the trigger enables the WIN SCP to process the call termination for the next available radio channel.

The WIN SCP requires a set of capabilities for PAS functionality. It is envisioned that the WIN SCP will primarily perform the priority and authorization validation of the NS/EP user and queue management of PAS calls. However, the capabilities of the WIN SCP may be expanded to provide other types of PAS functionality to an NS/EP user. Such capabilities include:
- Serving the PAS call queue by repeatedly attempting to acquire a radio channel for the first call in queue at regular intervals until served, or initiating call service based on MSC notification of a radio channel becoming available.

- Managing the queues on a per cell basis by priority level and time of arrival.

- Managing the queues to give priority to calls terminating at the served MS over calls originating from the served MS, or vice versa, or either by priority.

- Enhancing the queue management to permit updating the cell queues to reflect NS/EP user or NS/EP called party movement between cells while the call is in queue as reported by the MSC in page responses to the page requests.

- Recognizing duplicate "No Radio Available" queries from the same call and ignoring the second request.

- Recognizing a different destination number (DN) request from the same MS and deleting the first request from the queue.

- Translating selected prefixes to specific DNs.

- Performing PIN validation, if implemented.

\section{POTENTIAL IMPLEMENTATION APPROACHES}

There are currently two implementation approaches that have been presented to the TIA standards committees, which will make a recommendation on the preferred method. Some of the specifics of these approaches are subject to change. Approach 1 was prototyped by the OMNCS and demonstrated to industry in 1999 using an MSC and lab facilities of a major MSC vendor. Approach 2 is a modification of Approach 1 and in most instances reduces the messaging overhead for the queuing process. Each approach is discussed briefly in this section.

Approach 1, Repeated Attempts, employs a repeated attempt approach to enable a queuing process in which queued PAS calls are assured of getting access to the next available radio channel in the cell before non-PAS calls. At Call Origination, this approach uses the new No_Radio_Available trigger and the new Request Radio Channel, Channel Reservation, and WIN Initiate Call capabilities. The Initiate Call capability in the WIN SCP improves radio channel access attempt performance because it is able to address MSs that return to an idle state before a radio channel becomes available. At Call Termination, this approach uses the No_Radio_Available trigger, the Request Radio Channel 
capability, and the Channel Reservation capability in conjunction with the normal call termination paging process. This approach queues arriving calls and grants the next available radio channel.

Approach 2, MSC Radio Channel Available Notification, requires augmenting the Channel Reservation capability to indicate the number of channels needed in the cell for queued calls, and having the MSC notify the WIN SCP when a radio channel becomes available in the cell. The advantage of this approach is that by knowing the number of channels required, the MSC can limit the impact of radio channel reservation. Furthermore, by notifying the $\mathrm{SCP}$ when a radio channel becomes available, the MSC can minimize the holding interval for the reservation of channels. At Call Origination, this approach also uses the No_Radio_Available trigger, the Request Radio Channel, Channel Reservation, and WIN Initiate Call capabilities. It is a queuing process in which queued PAS calls are assured of getting access to the next available radio channels in the cell before non-PAS calls. The coupling of the Channel Reservation capability with the MSC Radio Channel Available Notification capability eliminates the need to repeat attempts as is done in Approach 1 (except in cases where mobility during the queuing delay has put the MS in a different congested cell). At Call Termination, this approach uses the No_Radio_Available trigger, the Request Radio Channel, Channel Reservation, and WIN Initiate Call capabilities.

This notification enhancement to the channel reservation capability virtually eliminates the need for the repeated attempts process used in Approach 1 and generally reduces the messaging overhead for the queuing process, while still providing the NS/EP user a high probability of radio channel access. However, the channel available notification approach may require air interface technology-dependent development within the MSC whereas Approach 1 does not. This is because the current technology-independent call processing software in an MSC usually interacts with the technologydependent software via requests for channel assignments. There currently is no provision for the technology-dependent software to provide self-initiated reporting to the technologyindependent software when channels become available.

\section{REFERENCES}

1. American National Standard, Signaling System No. 7 (SS7) - High Probability of Completion (HPC) Network Capability, ANSI T1.631-1993, June 28, 1993.
2. J. Szeto, C. Krafthofer, and P. V. McGregor, "Benefit of Higher Priority for National Security / Emergency Preparedness Calls in Signaling System Number 7," OPNETWORK 99, September 1999.

3. Federal Communications Commission, 00-242, "In the Matter of the Development of Operational, Technical and Spectrum Requirements for Meeting Federal, State and Local Public Safety Agency Communication Requirements Through the Year 2010 - Establishment of Rules and Requirements for Priority Access Service," WT Docket No. 96-86, Second Report and Order, Adopted July 3, 2000.

4. Telecommunications Industry Association (TIA), Wireless Intelligent Network, TIA/EIA/IS-771, July 1999. 